\title{
CONVÊNIOS COMO MECANISMO DE RELACIONAMENTO ENTRE UNIVERSIDADE E SOCIEDADE NO BRASIL: O CASO DA UNIVERSIDADE ESTADUAL DE CAMPINAS*
}

\author{
Cláudia NaOmi Sakashita ${ }^{1}$ id \\ André Sica de CAMPOS ${ }^{2}$ (D) \\ Salvador Antonio Mireles Sandoval ${ }^{3}$ (1) \\ Liz Felix Greco ${ }^{4}$ (D)
}

\begin{abstract}
RESUMO: Desde os anos 1990 há aproximação entre universidades e sociedade. Nesse contexto, buscou-se entender como essa aproximação se manifesta em convênios. Realizou-se levantamento de dados primários dos convênios da Universidade Estadual de Campinas (Unicamp), entre 2000 e 2012, celebrados com entes externos. Os resultados indicam sua frequência e intensidade, os tipos de organização envolvidos, sua duração, seus valores e sua natureza, de acordo com as unidades da Unicamp. De um lado, os convênios duram, em média, 30 meses, o que permite, em princípio, o reforço dos laços de cooperação entre a Unicamp e seus conveniados. De outro, verifica-se uma concentração em recursos financeiros e entes a partir do setor público, reforçando a relevância dessa fonte de recursos para a universidade.
\end{abstract}

Palavras-chave: Parcerias universidade-sociedade. Políticas. Missão.

\section{CONTRACTUAL MECHANISMS LINKING UNIVERSITY AND SOCIETY IN BRAZIL: THE CASE OF STATE UNIVERSITY OF CAMPINAS}

ABSTRACT: Since the 1990s there has been closer ties between universities and society. This article seeks to understand this phenomenon in terms of contracts. We conducted primary data collection based on

\footnotetext{
*Esta investigaçáo contou com o apoio da agência de fomento à pesquisa CAPES (Coordenaçáo de Aperfeiçoamento de Pessoal de Nível Superior). Este artigo é resultado de projeto de Tese de Doutorado (2016) intitulado $A$ interação entre universidade e usuários do conhecimento e as funçôes de ensino e pesquisa: um estudo de caso múltiplo sobre a Unicamp.

${ }^{1}$ Universidade Estadual de Campinas - Campinas (SP), Brasil. E-mail: claumagi@gmail.com

${ }^{2}$ Universidade Estadual de Campinas, Faculdade de Ciências Aplicadas - Campinas (SP), Brasil.

E-mail: andre.campos@fca.unicamp.br

${ }^{3}$ Pontifícia Universidade Católica de São Paulo - São Paulo (SP), Brasil. E-mail: ams1910@gmail.com ${ }^{4}$ Universidade Estadual de Campinas, Instituto de Geociências, Programa de Pós-Graduação em Política Científica Tecnológica - Campinas (SP), Brasil. E-mail: felixgrecoliz@gmail.com DOI: 10.1590/ES0101-73302019188572
} 
contracts between Universidade Estadual de Campinas (Unicamp) and external entities (2000-2012). The results indicate their frequency, intensity, organizations involved, their duration, the values involved and their nature, according to Unicamp units. This data indicates that, on average, the agreements lasted 30 months, strengthening ties of cooperation between Unicamp and external entities. We also observed a concentration of financial resources from public sector agencies, reinforcing the importance of this source to the university.

Keywords: University-society partnerships. Policies. Mission.

\title{
CONVENIOS COMO MECANISMO DE RELACIÓN ENTRE UNIVERSIDAD Y SOCIEDAD EN BRASIL: EL CASO DE LA UNIVERSIDAD ESTADUAL DE CAMPINAS
}

\begin{abstract}
RESUMEN: Desde los años 1990 hay aproximación entre universidades y sociedad. En ese contexto, se buscó entender cómo esa aproximación se manifiesta en convenios. Se realizó levantamiento de datos primarios de los convenios de la Universidad Estadual de Campinas (Unicamp), entre 2000 y 2012, celebrados con entes externos. Los resultados indican su frecuencia e intensidad, los tipos de organización involucrados, su duración, sus valores y su naturaleza, de acuerdo con las unidades de la Unicamp. De un lado, los convenios duran, en promedio, 30 meses, lo que permite, en principio, el refuerzo de los lazos de cooperación entre la Unicamp y sus conveniados. De otro, se verifica una concentración en recursos financieros y entes a partir del sector público, reforzando la relevancia de esa fuente de recursos para la universidad.
\end{abstract}

Palabras clave: Asociaciones universidad-sociedad. Políticas. Misión.

\section{Introdução}

A

partir dos anos de 1990, limites aos recursos públicos aportados nas universidades públicas de pesquisa impulsionaram a aproximaçáo entre governo, empresa e universidade (G-E-U) — por exemplo, por meio de convênios com financiamento alternativo aos conselhos de pesquisa (MOLLAS-GALLART et al., 2002). Nesse quadro, o argumento da Hélice Tripla (HT) propóe explorar a sinergia entre os três atores, gerando uma interação dinâmica que propicia a inovação e, quando relevante, a sobreposição virtuosa entre eles. Entretanto, essa teoria reflete a maturidade dos atores presente nos países desenvolvidos (ETZKOWITZ \& LEYDESDORFF, 2000). Nessa realidade, a universidade oferece mão de obra e conhecimento aplicado para seu entorno socioeconômico com frequência, com clara existência de demanda social e empresarial por seu conhecimento; fontes de financiamento privado; grande oferta de mão de obra qualificada; e legislação mais desburocratizada (CAMPOS, 2010; 
PAVITT, 2001; DAGNINO \& GOMES, 2001). Esses fatores nem sempre são disponíveis no caso do Brasil e, mais especificamente, da Unicamp.

Nesse contexto, sabendo-se das limitações para a vinculação entre a Unicamp e seu meio externo, busca-se o encaminhamento da seguinte questão de pesquisa: quem são e com que frequência entes externos se vinculam à Unicamp por meio de convênios? A fim de responder a essa questão, este estudo investigou a evoluçáo dos esforços da Unicamp em se conectar com a sociedade por meio de convênios $^{1}$ ocorridos entre os anos 2000 e 2012 quanto a:

- natureza e tipo de usuário do conhecimento conveniado;

- $\quad$ frequência e duração dos convênios;

- $\quad$ natureza dos recursos pertinentes;

- $\quad$ valores envolvidos;

- $\quad$ quantidade de eventos por ano e por centro de custo.

Para tanto, na seção seguinte, revisa-se a literatura sobre a relação G-E-U. Na metodologia, explica-se como o estudo foi realizado. Em seguida, os resultados quantitativos e, finalmente, conclui-se o artigo.

\section{Revisão da literatura}

\section{Relação com a sociedade: a terceira missão da universidade}

Os Estados Unidos foram os precursores na terceira missão da universidade. Uma política de doação de terras pelo Governo Federal (Morrill Act de 1862) redundou em escolas de agricultura e engenharia ocupadas com atividades de ensino e pesquisa — inclusive abertas à comunidade — , inaugurando assim a função de extensão à sua população (RASMUNSSEN, 1989). Mais recentemente, após 1990 , esse papel passou a ser reavaliado, com ênfase na participação das universidades de forma mais ativa no processo de desenvolvimento e na relaçáo com a sociedade e as empresas. Esse novo papel é chamado de terceira missão da universidade; junto às missóes de ensino e pesquisa formam a segunda revolução acadêmica (ETZKOWITZ \& LEYDESDORFF, 2000). Essa relação estabeleceu-se para complementar a obtenção de recursos públicos para pesquisa, legitimando a universidade junto à sociedade — quanto às demandas sociais e das empresas por conhecimento.

Nesse contexto, espera-se que as universidades possam contribuir para o desenvolvimento regional por meio da sua interação com usuários do conhecimento (MOLLAS-GALLART et al., 2002). Para Etzkowitz e Leydesdorff (2000), a pesquisa universitária legitimada é aquela que obtém aplicação econômica, o que 
deve ser combinada com a capacidade de incorporar novas disciplinas e áreas de pesquisa socialmente relevantes.

Todavia, o sucesso econômico não depende apenas da atuaçáo e do posicionamento das universidades, e sim de um conjunto de elementos, como a infraestrutura de ambas as partes, as políticas públicas, as leis, a disponibilidade financeira, a qualificação pessoal, os aspectos sócio-históricos e culturais. Mowery e Sampat (2004) alertam para a necessidade de se aprofundar instrumentos e mecanismos para compreensão da terceira missão. Caso essa missão se dê com o enfraquecimento do compromisso dos acadêmicos com ensino e pesquisa, tais esforços podem ser contraproducentes para o desenvolvimento econômico.

O estabelecimento da identidade e dos papéis da universidade é questão antiga, havendo a autocrítica e o questionamento dos próprios acadêmicos. Ainda sobre a terceira missão, Kerr (1972) aponta que Flexner, na década de 1930, denunciava a perda da identidade universitária com sua crescente semelhança com outras organizaçóes, como escolas secundárias, vocacionais e de formação de professores, desvirtuando a missão precípua de ampliar o conhecimento.

No Brasil, desde os anos 1990 existe a redução das atividades estatais por meio das privatizaçóes. Por outro lado, fortaleceram-se os programas de pós-graduação e de fusão de ensino e pesquisa, valorizando recursos humanos e a possibilidade de formação de redes formais e informais a partir da aproximação das empresas via atividades de pesquisa colaborativa em áreas como saúde coletiva, agricultura, petróleo, mineração, metalurgia e aeronáutica (SUZIGAN \& ALBUQUERQUE, 2011; CAMPOS, 2010). Nos anos 2000, inúmeras tentativas de fomentar a relação universidade-empresa foram realizadas por políticas de aproximação entre as partes (SUZIGAN \& ALBUQUERQUE, 2011). Todavia, é de grande relevância a participação do setor público na execução e no financiamento à pesquisa e ao ensino universitário, o que denota a limitaçáo dessas dinâmicas de contribuição ao desenvolvimento regional no Brasil, se mensurado pelo aporte de vínculos da universidade com o setor privado (CAMPOS, 2010).

\section{Hélice Tripla: teoria da interação entre universidade e sociedade}

Da literatura disponível, selecionamos a Teoria da HT, um marco conceitual que discorre sobre a vinculação entre universidade e usuários do conhecimento. A partir dos anos 1990, uma crescente e sinérgica interação entre G-E-U se estabeleceu e com ela um argumento explicativo desta interaçáo, a HT. No primeiro modelo, conhecido como HT 1 ou Triângulo de Sábato ${ }^{2}$, de Sábato e Botana (2011), G-E-U se relacionam de modo estático — o Estado atuando como ente dominador da relação. Tal modelo (Figura 1), comumente aplicado no 
sistema político-econômico socialista, sofre adaptação, mais branda, nos países da América Latina (ETZKOWITZ \& LEYDESDORFF, 2000).

A segunda versão do modelo de interação entre G-E-U é chamada Laissez-faire e, apesar de tal nome sugerir liberdade ou ausência de regras, consiste em delimitar as esferas de atuação de cada ente, estabelecendo fronteiras rígidas entre eles (Figura 2).

Embora menos difundido que os dois primeiros modelos, o modelo HT 3 (Figura 3), proposto por Etzkowitz e Leydesdorff (2000), é mais funcional, por causa do seu dinamismo e da sincronia promovida entre os entes, propiciando a inovaçáo, visto como base do crescimento econômico. Nesse modelo, as três esferas G-E-U se sobrepóem para gerar conhecimento, resultando na formação de uma organização híbrida que faz emergir outras interfaces. Não há estabilidade na relação, já que a comunicação e as expectativas dos atores guiam e reconstroem os arranjos institucionais que convergem para o atendimento das metas em comum; em outras palavras, cada sistema seria definido de acordo com cada projeto de

\section{Figura 1}

Modelo Hélice Tripla 1 ou Triângulo de Sábato.

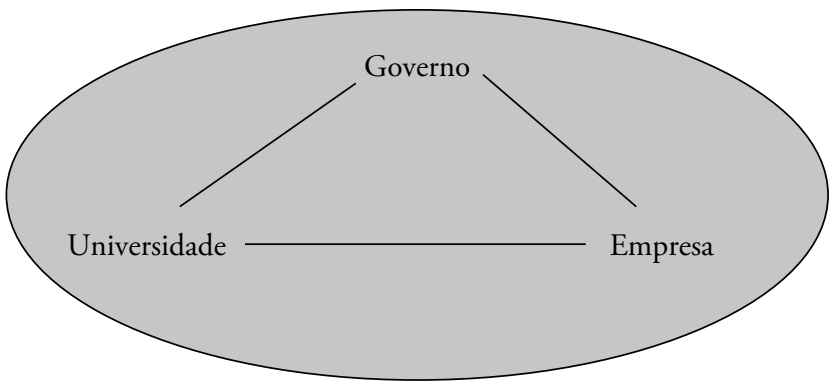

Fonte: Etzkowitz \& Leydesdorff (2000, p. 111).

Figura 2

Modelo Laissez-faire.

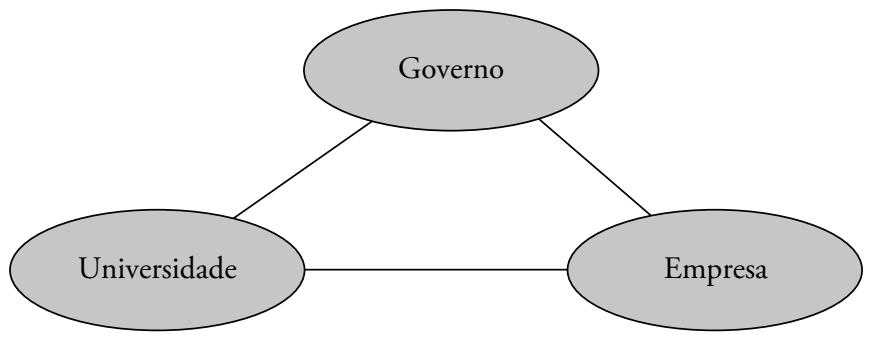

Fonte: Etzkowitz \& Leydesdorff (2000, p. 111). 
pesquisa. $\mathrm{O}$ modelo promove náo só o relacionamento entre G-E-U, mas também uma transformação interna em cada uma dessas esferas (ETZKOWITZ \& LEYDESDORFF, 2000).

Dessa maneira, na HT 3, o arranjo institucional propóe dinamismo e tridimensionalidade como em um "DNA" (para dar ideia de movimento, contrária à estabilidade e à rigidez do Triângulo de Sábato e do modelo Laissez-faire); além da instabilidade, cada hélice está relacionada às outras duas, havendo assim a possibilidade de sobreposição de comunicação, de redes de relacionamento e de organizaçáo entre os atores, os quais se reorganizam e se harmonizam em infraestrutura que converge em metas comuns.

Contudo, essa visão não está isenta de contra-argumentos; entre eles, Florida (1999) menciona que a universidade é necessária para o crescimento econômico, porém não deve ser responsável por ele. Dessa forma, a ela caberia a formação de talentos, papel complementar no processo, mas essencial, já que, ainda de acordo com essa visão, uma população inteligente e crítica é fonte de recurso nobre para qualquer economia.

Desse modo, embora o autor afirme não ser contra a relação U-E, acredita que atribuir à ciência o papel de alavancar a economia por meio da inovação tecnológica é um equívoco, pois ela não opera como uma máquina, mas apenas como uma das peças da infraestrutura de produçáo de conhecimento, ou seja, atua na promoção de mecanismos para geração e aproveitamento de talentos (FLORIDA, 1999).

Essa teoria, portanto, apresenta modelo explicativo da interaçáo entre G-E-U nos países centrais, onde a vinculação é dinâmica e efetiva financeiramente, em incentivos políticos e em inovação. Realidade bem distinta, como é o caso da Unicamp, situada em país de capital periférico. A fim de esclarecer as condiçōes dessa relaçấo no caso da Unicamp, propóe-se a seguinte questão a nortear este artigo: quem são e com que frequência entes externos se vinculam à Unicamp por meio de convênios?

\section{Figura 3}

Modelo Hélice Tripla 3.

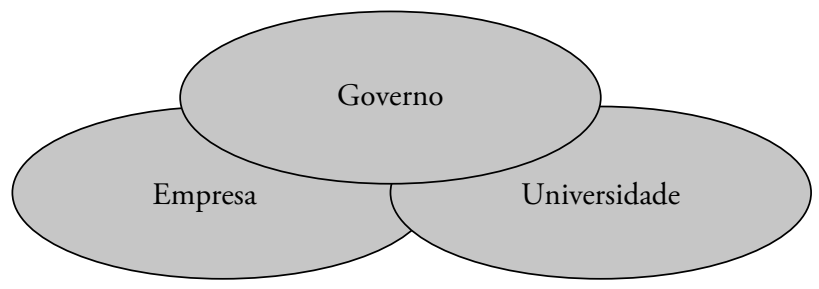

Fonte: Etzkowitz \& Leydesdorff (2000, p. 111). 


\section{Metodologia}

Para responder à pergunta desta pesquisa, buscamos dados quantitativos, com o recolhimento de dados de documentos primários e secundários da Unicamp e seus respectivos centros de custo, entre 2000 e 2012 - contendo neste levantamento as interaçóes entre a Unicamp e a sociedade, com intervenção administrativa da Fundação de Desenvolvimento da Unicamp (Funcamp) ${ }^{3}$.

A pesquisa de dados quantitativos consistiu em analisar o levantamento de dados quantitativos de todos os convênios celebrados entre os anos de 2000 a 2012 entre a Unicamp e as entidades externas em geral. Tal informação foi disponibilizada pela Diretoria Geral Administrativa $(D G A)^{4}$ e consiste em uma planilha (software Excel, Microsoft) de 194 páginas contendo: código; nome das partes envolvidas; objeto; número de registro; centro de custo da parte envolvida da Unicamp; datas de início e de término; executor/nome do docente que coordenou; ente externo que firmou essa parceria com a Unicamp; tipo e natureza da parceria; e valor financeiro envolvido, em reais. Com isso, pretende-se especificamente:

- identificar a natureza e o tipo dos usuários do conhecimento que interagem;

- levantar a frequência e a duração das incidências das vinculaçóes entre Unicamp e usuários do conhecimento;

- determinar valores envolvidos, os quais foram corrigidos anualmente de acordo com o Índice Nacional de Preços ao Consumidor Amplo (IPCA);

- mensurar quantidade de eventos por ano e por centro de custo.

Uma vez caracterizado o acumulado de 13 anos de relacionamento entre Unicamp e sociedade, qualificamos quantitativamente, de formas geral e detalha$\mathrm{da}$, analisando a incidência por faculdade e/ou institutos de ensino e pesquisa, por área de conhecimento, por grupo de gestão e por centros e núcleos.

Ficamos impossibilitados, porém, de aprofundar sobre o tipo/motivo do convênio - pesquisa, desenvolvimento de produto, curso, validação de tecnologia, consultoria ou serviço — , pois no relatório disponibilizado pela DGA há somente breve resumo sobre o objeto dos convênios, os quais estavam descritos, em sua maioria, com termos técnicos e específicos de cada área. Para interpretar tal dado, seria necessário o auxílio de um profissional de cada área, habilitado tecnicamente para discernimento dos termos utilizados. Além disso, a linguagem empregada nos relatórios disponibilizados não é padronizada (o objeto ora é disponibilizado de maneira descritiva e completa, ora abreviado ou com um "apelido"), as informaçóes são insuficientes (falta de legenda dos termos técnicos), há campos vazios ou incompletos (não descrição das finalidades, ou se pesquisa, serviços, ensino, acordo de cooperação científica ou consultoria), 
em códigos e siglas específicas de cada área. $\mathrm{O}$ modo de apresentação dos dados pela DGA não favoreceu melhor entendimento, análise e aprofundamento deles.

\section{Evidências das interações entre Universidade Estadual de Campinas e entes externos entre os anos 2000 e 2012}

\section{Quantidade de convênios}

A quantidade de convênios efetivados entre 2000 e 2012 chegou a um total de 5.136 (Gráfico 1). Entre os anos de 2000 e 2007, a incidência foi de crescimento contínuo, passando do patamar das 200 ocorrências por ano, em 2000 e 2001, para o das 300 ocorrências, em 2002 e 2003, chegando às 400 ocorrências anuais, entre 2004 e 2006. Atingiu o topo de crescimento em 2007, com 507 convênios, com ligeira queda para a casa dos 400 nos 2 anos seguintes, quando, finalmente, atingiu o patamar de 300 convênios nos três últimos anos.

A quantidade média de convênios foi de 395 por ano, no decorrer dos 13 anos analisados, e a sua realização aconteceu com relativa regularidade, não havendo variaçôes relevantes nos números, ao longo desse período.

\section{Natureza das fontes dos recursos recebidos}

Os valores recebidos pela Unicamp foram classificados como fontes de natureza pública, privada, mista e não informada/outras. As incidências (Gráfico 2)

\section{Gráfico 1}

Quantidade de convênios: Universidade Estadual de Campinas (Unicamp) versus sociedade, 2000 a 2012.

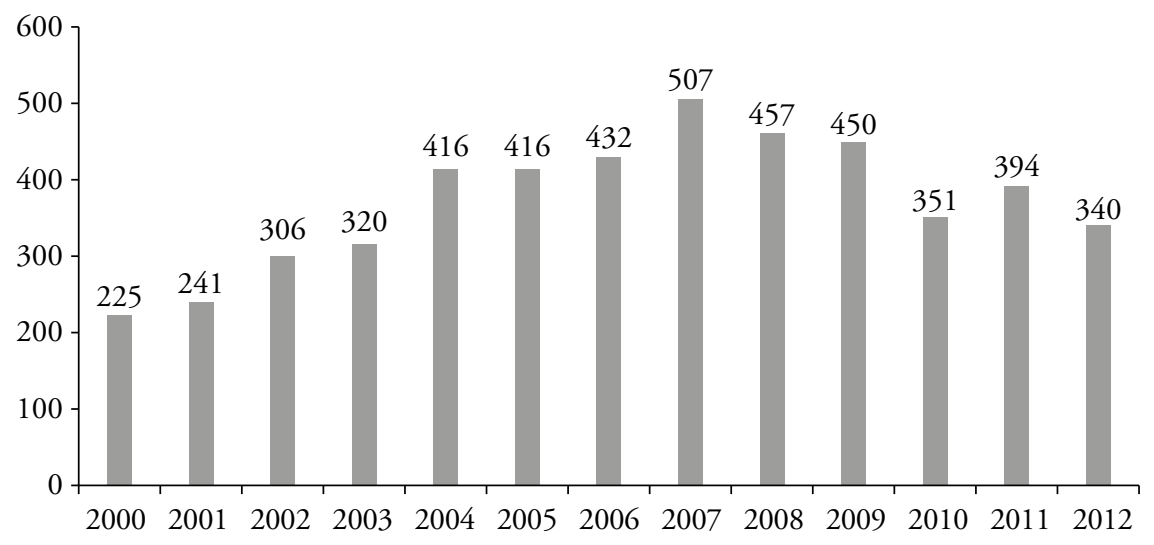

Fonte: dados da Diretoria Geral de Administração (DGA)/Unicamp, elaborado pelos autores. 
são discrepantes quando comparamos a fonte pública com as demais. Os recursos públicos representaram $88 \%$ do total de recursos recebidos, contra $9 \%$ dos recursos privados, $0,6 \%$ dos recursos mistos e $2 \%$ dos outros.

No acumulado dos 13 anos pesquisados (Gráfico 3), os recursos de origem pública obtiveram maior incidência, exceto no ano de 2003, em que contabilizaram

\section{Gráfico 2}

Fonte de recursos recebidos pela Universidade

Estadual de Campinas (Unicamp), em \%, 2000 a 2012.

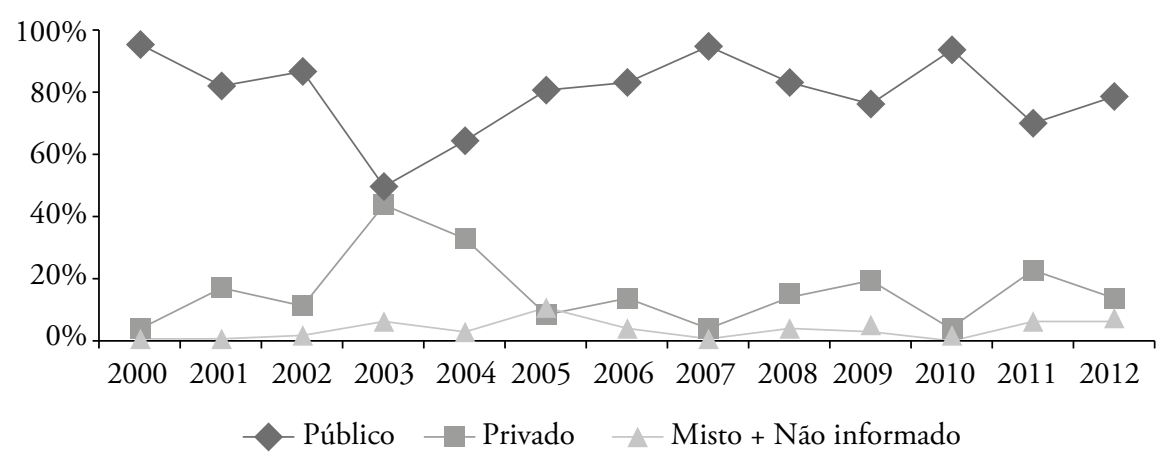

Fonte: dados da Diretoria Geral de Administração (DGA)/Unicamp, elaborado pelos autores.

\section{Gráfico 3}

Comparação entre fontes de recursos públicos e privados, em \%, 2000 a 2012.

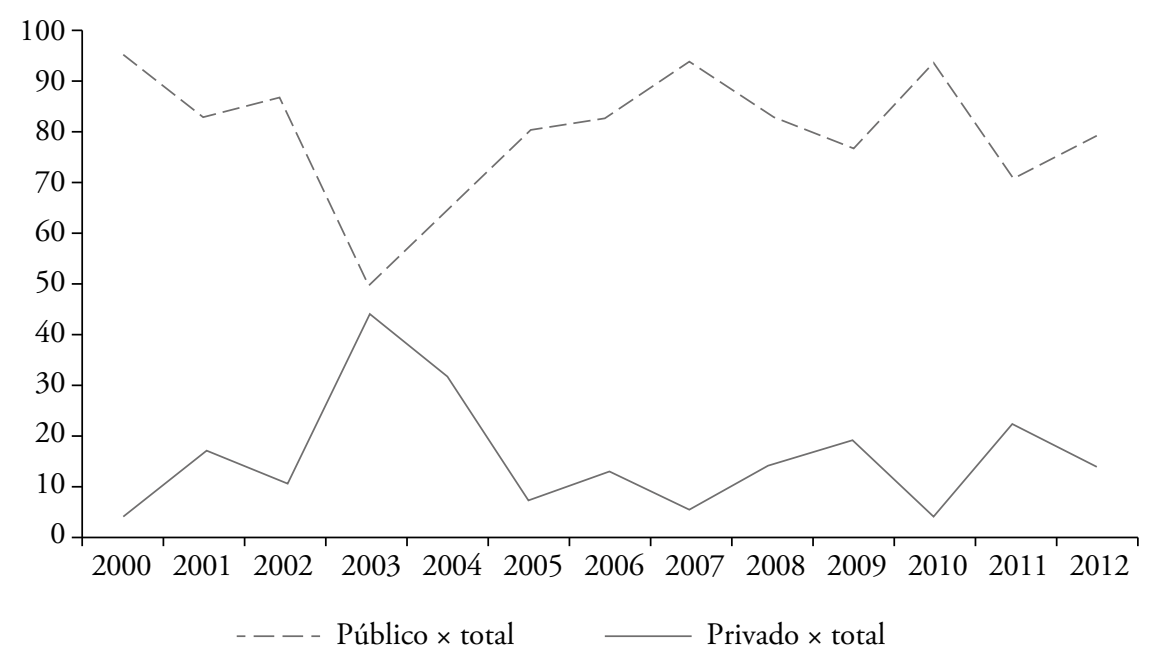

Fonte: dados da Diretoria Geral de Administração (DGA)/Universidade Estadual de Campinas (Unicamp), elaborado pelos autores. 
divisão de partes iguais dos valores, tanto da esfera pública como da privada, sendo esse ano a única incidência em que as porcentagens se aproximaram. Vale ressaltar que em 8 dos 13 anos analisados a porcentagem dos recursos públicos ultrapassou $80 \%$ do total.

\section{Quantidade de convênios e recursos financeiros recebidos por área de conhecimento}

A partir desse ponto, para efeito de análise, excluiremos os centros de custo referentes à área de saúde, por se tratar de área historicamente diferente dos demais institutos de ensino e pesquisa — tanto na incidência como nos tipos e modelos -; além do mais, essa área recebe recursos do Governo Federal, pelo Sistema Único de Saúde (SUS), referentes a procedimentos hospitalares, laboratoriais, farmacêuticos, entre outros, os quais destoam, no sentido de serem muito superiores aos demais.

Em se tratando de indicadores de quantidade de convênios versus quantidade de recursos financeiros recebidos por área de conhecimento (Gráfico 4), as áreas de gestâo (apoio administrativo, pró-reitorias e reitoria) foram as que mais se destacaram, com a realização de 1.251 convênios e com maior incidência no recebimento de recursos financeiros.

\section{Gráfico 4}

Quantidade de convênios e valores recebidos* (em milhóes de reais) por área de conhecimento. Acumulado 2000 a 2012.

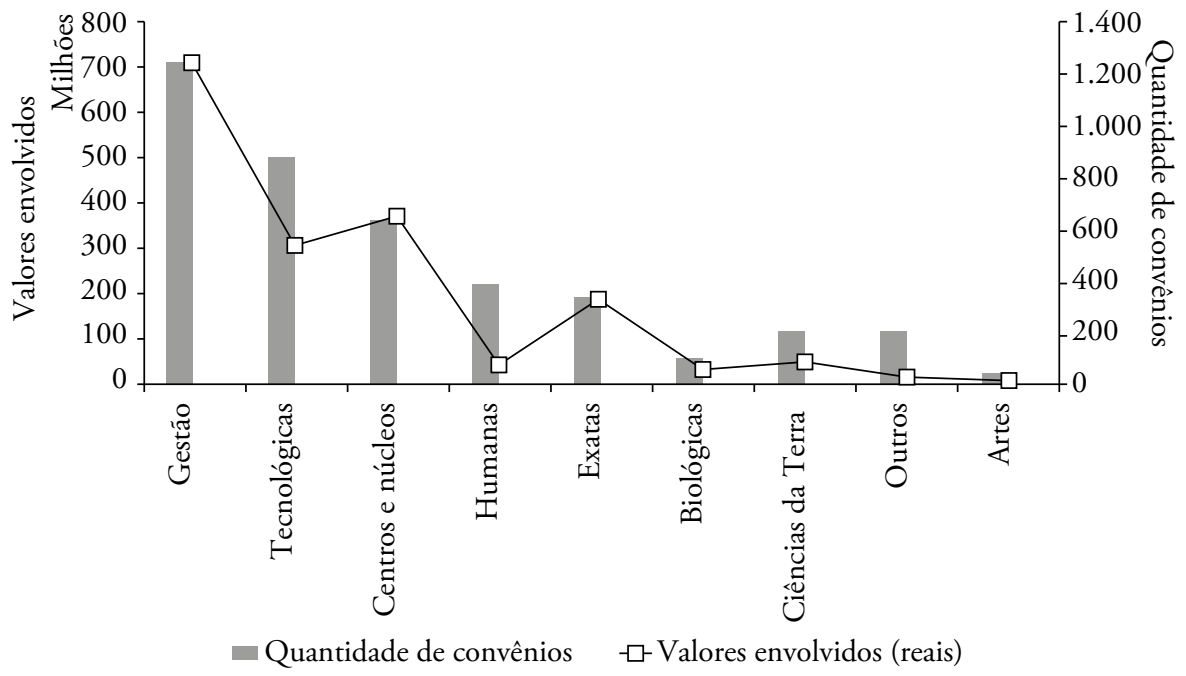

*Valores nominais inflacionados pelo Índice Nacional de Preços ao Consumidor Amplo (IPCA) até 2012.

Fonte: dados da Diretoria Geral de Administração (DGA)/Universidade Estadual de Campinas (Unicamp), elaborado pelos autores. 
Centros e núcleos e tecnológicas (Gráfico 4) destacaram-se nesses dois indicadores, sendo que o primeiro obteve o segundo maior valor financeiro (R \$ 370,5 milhóes, valores corrigidos pelo IPCA ${ }^{5,6}$ até 2012) e o terceiro em realização de convênios (639). Já a área de tecnológicas recebeu o terceiro maior valor financeiro ( $\mathrm{R}$ \$ 303,8 milhōes) e o segundo maior montante, com 882 ocorrências.

\section{Tipos de ente externo contratante por valores recebidos}

No levantamento sobre tipo de ente contratante (Gráfico 5) por montante de valores recebidos, destacam-se os institutos de apoio à pesquisa e as empresas públicas, que somaram $65 \%$ do total, confirmando assim o predomínio das receitas públicas levantadas anteriormente, nos Gráficos 3 e 4 . A indústria está na terceira colocação, em termos de recebimento de valores, tendo representado $15 \%$ do total das interaçóes.

\section{Quantidade de convênios, valores recebidos, duração média em meses no ensino e pesquisa}

Dos dez institutos de ensino e pesquisa com maior incidência de interação (Gráfico 6), cinco foram representantes da área de tecnológicas. Como já

\section{Gráfico 5}

Tipos de entidade contratante, em \%. Acumulado 2000 a 2012.

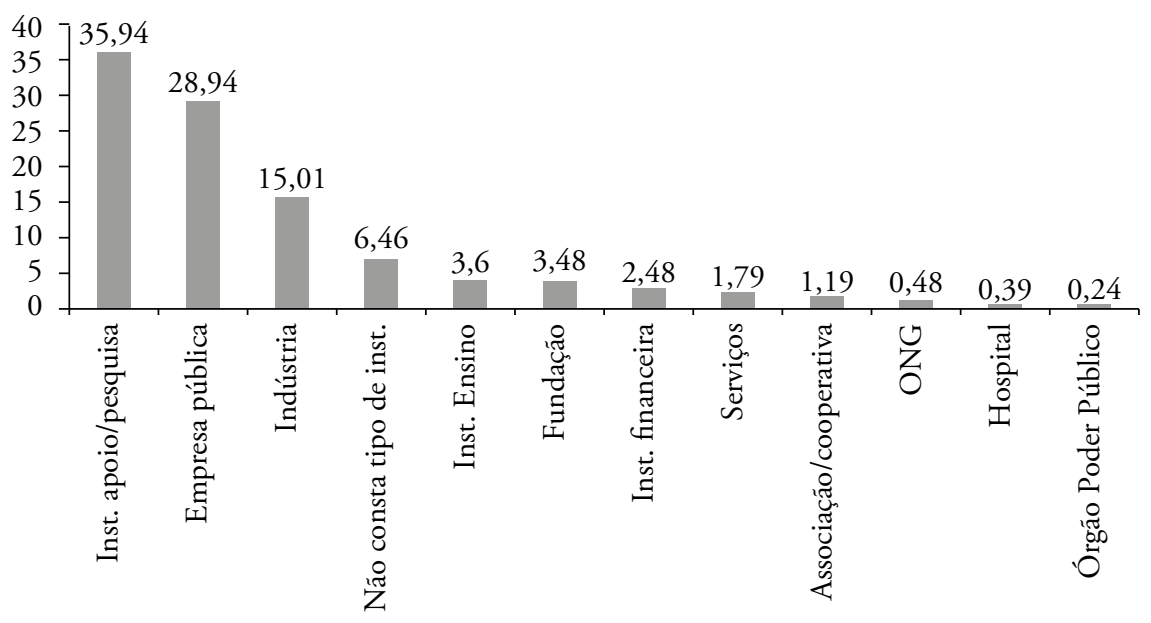

Inst.: instituição; ONG: organização não governamental.

Fonte: dados da Diretoria Geral de Administração (DGA)/Universidade Estadual de Campinas (Unicamp), elaborado pelos autores. 
esperado, as tecnológicas são as que mais interagem com o meio externo, tanto na quantidade de convênios realizados como em valores financeiros recebidos. Assim, esses cinco institutos somados (Faculdade de Engenharia Elétrica e Computação - FECC, Instituto de Química - IQ, Faculdade de Engenharia Mecânica - FEM, Faculdade de Engenharia Civil - FEQ, Arquitetura e Urbanismo da Unicamp e Centro Pluridisciplinar de Pesquisas Químicas, Biológicas e Agrícolas - CPQBA - este constitui um centro pluridisciplinar) representaram 64\% do total de valores recebidos pelos institutos de ensino e pesquisa. Esses cinco institutos representaram $41 \%$ do total. A FEEC está com larga vantagem, ocupando o primeiro lugar, com 348 convênios, seguida do IQ, com 174. O mesmo posicionamento se repete no recebimento de valores financeiros, com R \$ 173,3 milhóes e R \$ 123,4 milhóes, respectivamente. Em termos de recebimentos financeiros, a FEEC teve receita $40,4 \%$ superior ao segundo classificado, o IQ.

\section{Gráfico 6}

Valores recebidos* (em milhóes de reais), quantidade de convênios por instituto de ensino e pesquisa e duração média em meses. Acumulado 2000 a 2012.

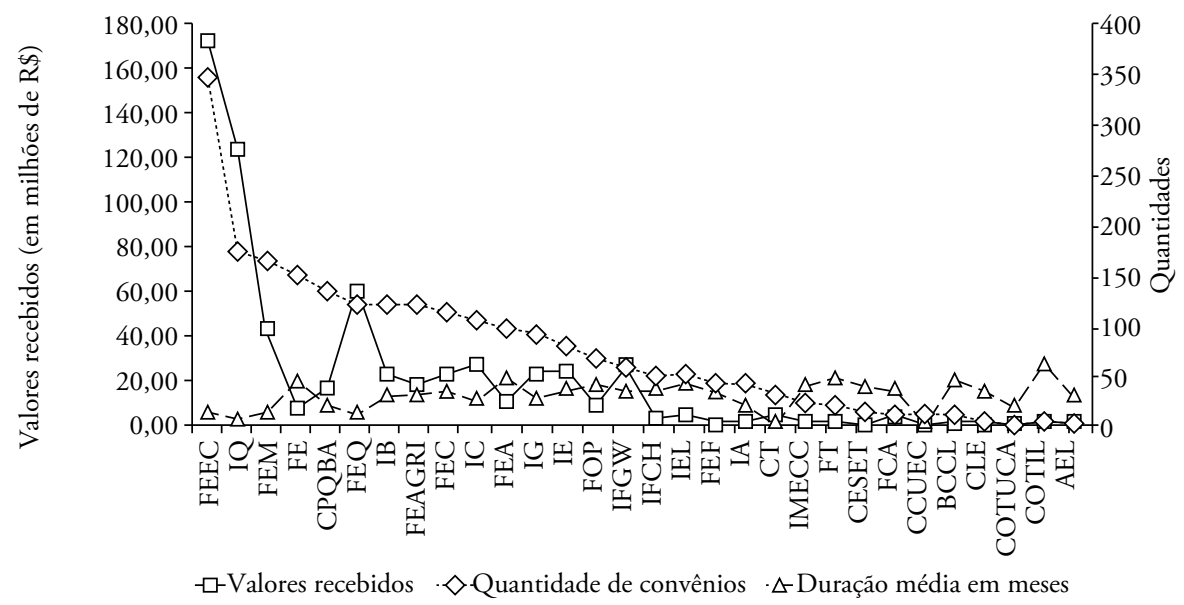

FEEC: Faculdade de Engenharia Elétrica e Computação; IQ: Instituto de Química; FEM: Faculdade de Engenharia Mecânica; FE: Faculdade de Educação; CPQBA: Centro Pluridisciplinar de Pesquisas Quimicas, Biológicas e Agricolas; FEQ: Faculdade de Engenharia Quimica; IB: Instituto de Biologia; FEAGRI: Faculdade de Engenharia Agricola da Universidade Estadual de Campinas (Unicamp); FEC: Faculdade de Engenharia Civil, Arquitetura e Urbanismo da Unicamp; IC: Instituto da Computação; FEA: Faculdade de Engenharia de Alimentos; IG: Instituto de Geociências; IE: Instituto de Economia; FOP: Faculdade de Odontologia de Piracicaba; IFGW: Instituto de Física Gleb Wataghin; IFCH: Instituto de Filosofia e Ciências Humanas; IEL: Instituto de Estudos de Linguagem; FEF: Faculdade de Educação Física; IA: Instituto de Artes; CT: Centro de Tecnologia; IMEEC: Instituto de Matemática, Estatística e Computação Científica da Unicamp; FT: Faculdade de Tecnologia; CESET: Centro Superior de Educação Tecnológica; FCA: Faculdade de Ciências Aplicadas; CCUEC: Centro de Computação da Unicamp; BCCL: Biblioteca Central Cesar Lattes; CLE: Centro de Lógica, Epistemologia e História da Ciência; COTUCA: Colégio Técnico de Campinas; COTIL: Colégio Técnico de Limeira; AEL: Arquivo Edgard Leuenroth; *valores nominais inflacionados pelo Índice Nacional de Preços ao Consumidor Amplo (IPCA) até 2012.

Fonte: dados da Diretoria Geral de Administração (DGA)/Unicamp, elaborado pelos autores. 
Já nos indicadores da média de duração dos convênios em meses (Gráfico 6), os dois primeiros institutos em quantidade de convênios e recebimento de valores financeiros, a FEEC e o IQ, obtiveram a média de duração de 11 e 6 meses, respectivamente. No caso da FEEC, foi $45 \%$ menor que a média de duração (20 meses) dos dez primeiros institutos. No caso do IQ, foi 70\% menor que a média dos mesmos dez primeiros.

Apontamos que uma das discrepâncias no Gráfico 6 de incidência sobre quantidade de convênios versus valores recebidos versus média de duração em meses se deu na Faculdade de Educação (FE), que obteve a quarta posiçâao em quantidade de convênios realizados (149) e a décima quinta em recebimento financeiro ( $\mathrm{R}$ \$ 7,4 milhóes).

A FE (Gráfico 6) também obteve a maior média de duração (44 meses) na realização de convênios entre os mesmos dez primeiros institutos, cuja média é de 20 meses de duraçáo. Uma das hipóteses de a média de duraçáo ser mais elevada se deve ao fato de a FE ofertar cursos, seja na forma de graduaçáo (inicial ou continuada de professores da rede pública de ensino), seja na de especialização, pós-graduação lato sensu ou outros, para professores e para a comunidade em geral.

A incidência de convênios na área de humanidades (Instituto de Estudos de Linguagem - IEL, Instituto de Filosofia e Ciências Humanas - IFCH, Faculdade de Educação Física - FEF — e Instituto de Economia - IE) ${ }^{7}$, excluindo-se a FE (Gráfico 6), foi baixa, com incidência de apenas quatro convênios por ano. Tal desempenho está dentro do esperado, levando-se em conta que os objetivos das interaçóes dizem mais respeito a consultorias e serviços do que desenvolvimento de técnicas e artefatos, em função da natureza do conhecimento em humanidades e ciências sociais dessas unidades.

\section{Quantidade de convênios, valores recebidos e duração média em meses nos centros e núcleos}

Abordaremos indicadores de quantidade de convênios versus valores recebidos versus média de sua duração nos centros e núcleos da Unicamp. O Centro de Estudos de Petróleo (CEPETRO) (Gráfico 7) obteve performance elevada na incidência de valores recebidos e quantidade de convênios. Ambos os indicadores apontam o primeiro lugar, com $\mathrm{R} \$ 222,3$ milhóes e 244 convênios, representando $59,99 \%$ do total de recursos recebidos por centros e núcleos e $38 \%$ do total realizado, respectivamente. Tratando-se de média de duração, esses indicadores encontram-se na média de duração desse grupo, que é de 38 meses.

$\mathrm{O}$ segundo destaque - bem abaixo da unidade anterior, CEPETRO vai para o Núcleo Interdisciplinar de Planejamento Energético (NIPE) (Gráfico 7), com realização de 121 convênios, quase metade do indicador citado anteriormente, e R\$ 70,1 milhões, 31,53\% do CEPETRO. 
As demais unidades obtiveram desempenho tímido (Gráfico 7), sendo que somente quatro (Centro de Pesquisas Meteorológicas e Climáticas Aplicadas à Agricultura - CEPAGRI, Núcleo de Estudos de Políticas Públicas - NEPP, Centro de Componentes Semicondutores - CCS - e Centro de Engenharia Biomédica - CEB) apresentaram recebimento de valores financeiros na casa dos R\$ 15 milhões. Assim também aconteceu com a quantidade de convênios firmados, sendo que 13 unidades, do total de 20 centros e núcleos, realizaram menos de 12 no decorrer dos 13 anos estudados, ou seja, menos de 1 por ano.

\section{Quantidade de convênios, valores recebidos e duração média em meses na área de gestão}

Notamos uma grande discrepância no primeiro órgão, Vice-Reitoria Executiva de Relaçóes Internacionais (VRERI) (Gráfico 8), que possui o maior

\section{Gráfico 7}

Quantidade de convênios, valores recebidos* (em milhóes de reais) e duração média em meses nos centros e núcleos. Acumulado 2000 a 2012.

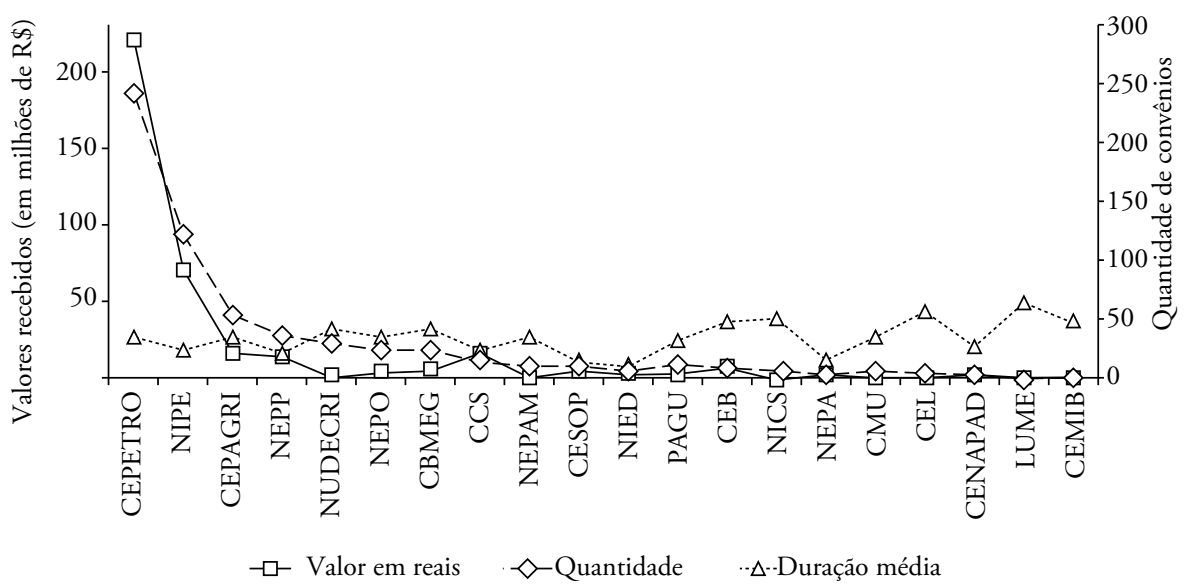

CEPETRO Centro de Estudos de Petróleo; NIPE: Núcleo Interdisciplinar de Planejamento Energético; CEPAGRI: Centro de Pesquisas Meteorológicas e Climáticas Aplicadas à Agricultura; NEPP: Núcleo de Estudos de Politicas Públicas; NUDECRI: Núcleo de Desenvolvimento da Criatividade; NEPO: Núcleo de Estudos de População "Elza Berquó"; CBMEG: Centro de Biologia Molecular e Engenharia Genética; CCS: Centro de Componentes Semicondutores; NEPAM: Núcleo de Estudos e Pesquisas Ambientais; CESOP: Centro de Estudos de Opinião Pública; NIED: Núcleo de Informática Aplicada à Educação; PAGU: Núcleo de Estudos de Gênero Pagu; CEB: Centro de Engenharia Biomédica; NICS: Núcleo Interdisciplinar de Comunicação Sonora; NEPA: Núcleo de Estudos e Pesquisas em Alimentação; CMU: Centro de Memória da Universidade Estadual de Campinas (Unicamp); CEL: Centro de Ensino de Linguas; CENAPAD: Centro Nacional de Processamento de Alto Desempenho; LUME: Núcleo Interdisciplinar de Pesquisas Teatrais da Unicamp; CEMIB: Centro Multidisciplinar para Investigação Biológica na Área da Ciência de Animais de Laboratório; *valores nominais inflacionados pelo Indice Nacional de Preços ao Consumidor Amplo (IPCA) até 2012.

Fonte: dados da Diretoria Geral de Administração (DGA)/Unicamp, elaborado pelos autores. 
número de convênios realizados (347) e é a oitava em recebimento de valores financeiros (R\$ 6 milhôes). Esses valores representam 28 e 1\%, respectivamente, das quantidades totais envolvidas na área de gestão. A hipótese dessa discrepância é a de se tratar de um órgão promotor de intercâmbio e troca de conhecimento entre pesquisadores (alunos, professores, grupos de estudo e trabalho), a qual intermedeia inúmeros acordos de cooperação científica e cultural, quando os valores, na sua maioria, destinam-se aos bolsistas contemplados nos projetos.

Inversamente proporcional à VRERI está a Pró-Reitoria de Pós-Graduação (PRPG) (Gráfico 8), com alta incidência de recebimento de recursos financeiros, obtendo a primeira classificação, com R \$ 378,2 milhōes. Tais recursos representaram $53 \%$ do total de recursos recebidos. Por outro lado, a quantidade de convênios celebrados correspondeu a 3\% do total.

No quesito tempo médio de duração (Gráfico 8), em meses, a incidência foi de 37 meses, tendo a Inova destoado desse patamar, com duração média de 99 meses.

\section{Gráfico 8}

Valores recebidos* (em milhôes de reais), quantidade de convênios e duração média em meses na área de gestão. Acumulado 2000 a 2012.

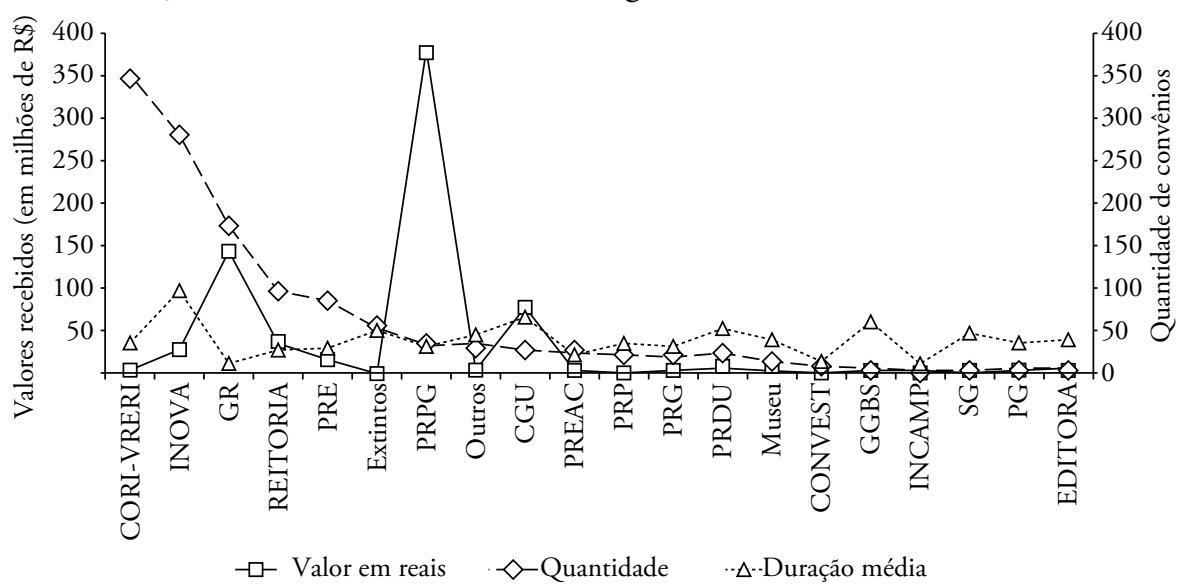

CORI-VRERI: Vice-Reitoria de Relações Internacionais; INOVA: Agência de Inovação da Universidade Estadual de Campinas (Unicamp); GR: gabinete do reitor; PRE: Pró-Reitoria de Extensão; PRPG: Pró-Reitoria de Pós-Graduação; CGU: Coordenadoria Geral da Universidade; PREAC: Pró-Reitoria de Extensão e Assuntos Comunitários; PRP: Pró-Reitoria de Pesquisa; PRG: Pró-Reitoria de Graduação;

PRDU: Pró-Reitora de Desenvolvimento Universitário; CONVEST: Comissão Permanente para os Vestibulares; GGBS: Grupo Gestor de Beneficios Sociais; INCAMP: Incubadora de Empresas de Base Tecnológica da Unicamp; SG: Secretaria Geral; PG: Procuradoria Geral; *valores nominais inflacionados pelo Índice Nacional de Preços ao Consumidor Amplo (IPCA) até 2012.

Fonte: dados da Diretoria Geral de Administração (DGA)/Unicamp, elaborado pelos autores. 


\section{Conclusões}

A ciência tem se organizado para atender tanto a demandas práticas quanto teóricas, definidas pela época, pelo local e pela cultura em que estão inseridas. Aqui tratamos da terceira missão da universidade, que é a de promover e alavancar a economia e gerar riquezas para a sociedade. Vimos, a partir dos dados apresentados, que a Unicamp apresenta relevante vinculação com entes externos por meio de convênios. Dada essa aproximação com usuários, a indústria - o ente mais almejado e que traria mais possibilidades de aprimoramento tecnológico de acordo com a HT - aparece como um dos principais entes externos conveniados com a Unicamp, ainda que em menor medida que entes do setor público. Por outro lado, com esse contato, há grande possibilidade de inovação.

Para Etzkowitz \& Leydesdorff (2000), o modelo de interação entre academia e sociedade deve ser dinâmico e de livre funcionamento, no qual, de acordo com cada situação e projeto, pode haver a predominância de uma das "hélices" (G-E-U) sobre as demais, mas sempre em uma relação sinérgica. Para os autores, tal modelo potencializaria a ação de cada membro envolvido nessa interação.

Diante dessa teoria, é possível dizer que o modelo adotado pela Unicamp está aquém do ideal proposto, uma vez que o ente mais atuante da interação é o setor público, ou seja, uma aproximação do Estado, dominante na intensidade, na frequência e nos valores financeiros envolvidos nos convênios. Assim, nossa análise não atende plenamente aos argumentos teóricos da HT, pois não é uma interação sinérgica, em que há a ação conjunta de forças simultâneas.

Em tempos de instabilidade econômica, o Estado apresenta menos condiçóes de alavancar a relação entre a universidade e a sociedade. Ademais, a esfera privada historicamente não é atraída para alavancar a pesquisa universitária no Brasil. Diante desses fatos, ressalta-se que nos 13 anos estudados, a média das receitas angariadas de origem privada foi de apenas $9 \%$ do total, contra $88 \%$ de recurso público, enquanto a média das verbas advindas da indústria, no mesmo período, equivaleram a $15 \%$ do total.

Como argumento justificativo da interatividade entre universidade e meio externo no Brasil, Suzigan e Albuquerque (2011) apontam que a tardia e problemática implantação do Sistema Nacional de Inovação (SNI) se deu por fatores históricos, como o modelo de economia agroexportador, que demandava baixas soluções tecnológicas, estabelecendo, portanto, uma infraestrutura científica pouco desafiadora. Somado à deficiência histórica na educação e na formação de mão de obra técnica, o sistema financeiro voltado ao crédito comercial de curto prazo não foi também favorável ao desenvolvimento adequado do SNI. Assim, a relação entre universidades e o setor industrial poderia ser mais relevante do que é atualmente. No caso do Brasil, uma das hipóteses levantadas por Suzigan e Albu- 
querque (2011) é a de que a criação tardia do sistema de universidades e institutos de pesquisa, aliada à industrialização tardia, são fatores limitadores do desenvolvimento. Além disso, a atual capitalização financeira global impacta e desfavorece os países que estấo à margem do capital, que, por sua vez, não investem em industrialização nem em geraçóes de empregos.

É nesse contexto que se encontra nosso levantamento empírico dos convênios realizados entre a Unicamp e os usuários do conhecimento entre 2000 e 2012. Notou-se que a duração foi, em média, de 30 meses, evidenciando que há uma tendência de realização de pesquisas de médio prazo. Entretanto, há inconstância no volume e em valores anuais. Entre os anos de menor e maior valor movimentado há uma diferença maior do que $1.700 \%$, ou seja, o montante de dinheiro mobilizado é irregular. Percebemos, ademais, que a maior parte dos recursos aportados na Unicamp adveio do setor público.

E também, como confirmado pelos indicadores quantitativos deste estudo, a área de tecnologia, por sua vocação "desenvolvimentista" desde sua fundação, sempre demandou maior montante de relacionamento com a sociedade; enquanto isso, a interação com a área de humanidades, instituída para criticar, sugerir e pensar sobre a sociedade, é pouco demandada. Embora esses resultados fossem esperados, perguntamos o porquê de não haver demanda por parte da sociedade de pesquisas da área de humanidades. Tal pergunta fica registrada para futura análise. Ademais, observamos que, dada a limitação dos dados, não é possível identificar a natureza do convênio celebrado. Dessa forma, os dados levantados para este estudo podem ser objeto de uma análise mais aprofundada em estudos futuros, para se elucidar a natureza das atividades envolvidas no convênio, o que não está evidenciado nos dados brutos coletados. De forma análoga, a Unicamp, a título de implicações práticas de nosso estudo, deve dispensar um tratamento mais aprofundado e cuidadoso dos dados relativos a convênios, no que tange ao tipo, ao objeto e à finalidade, de sorte a facilitar tanto o monitoramento como a avaliação deles quanto à natureza das atividades efetuadas.

Como fatores limitadores desta pesquisa, elegemos os dados, especificamente "tipo/motivo" (o objeto do contrato, em termos administrativos e jurídicos), para realização dos convênios, informações essas faltantes no relatório disponibilizado pela DGA — Unicamp.

\section{Notas}

1. Definição de convênio, cf. Instrução DGA, no 5 de 11 de julho de 2001: "Acordo firmado por entidades públicas de qualquer espécie, ou entre estas e organizaçôes particulares, para realização de objetivos de interesse comum dos partícipes" (UNICAMP, 2011). 
2. Teoria de 1968, os atores Estado, Empresa e Universidade seriam os responsáveis pela promoção do desenvolvimento socioeconômico, via incentivo científico-tecnológico, sendo o Estado o articulador central, promotor de políticas e recursos dessa relação triangular.

3. É uma fundação privada, criada em 1977, com o intuito de atender às demandas de pesquisa, ensino e extensão, sob Escritura de instituição no livro 286, folha 053, em 31 de maio de 1977, no $4^{\circ}$ Tabeliáo de Notas da Comarca de Campinas, São Paulo. Para mais detalhes sobre a fundaçáo, vide Sakashita (2011).

4. Solicitado por meio de abertura de protocolo do Serviço de Informação ao Cidadão (SIC), responsável por toda e qualquer informação referente à Unicamp, por meio do site http://www. unicamp.br/unicamp/sic-unicamp.

5. Índice Nacional de Preços ao Consumidor Amplo (IPCA): é um índice de inflação medido "nas regióes metropolitanas de Belém, Fortaleza, Recife, Salvador, Belo Horizonte, Rio de Janeiro, São Paulo, Curitiba, Vitória e Porto Alegre, Brasília e municípios de Goiânia e Campo Grande”. "Abrange as famílias com rendimentos mensais compreendidos entre 1 e 40 salários-mínimos, qualquer que seja a fonte de rendimentos, e residentes nas áreas urbanas das regiōes”. Sua série histórica inicia em fevereiro de 1980" (IBGE, 2018).

6. Todos os valores financeiros citados no decorrer do texto foram corrigidos pelo IPCA até ano de 2012.

7. Se incluirmos o Arquivo Edgard Leuenroth (AEL), a Biblioteca Central Cesar Lattes (BCCL) e o Centro de Lógica, Epistemologia e História da Ciência (CLE), que são órgãos de apoio da área de ensino e pesquisa, esse indicador diminuiria para uma média de dois convênios por ano.

\section{Referências}

CAMPOS, A. A review of the influence of long-term patterns in research and technological development (R\&D) formalization on university-industry links. Revista Brasileira de Inovaçáo, v. 9, p. 379-410, 2010.

DAGNINO, R.; GOMES, E. A relação universidade-empresa: comentários sobre um caso atípico. Gestáo e Produçáo, v. 10, p. 283-292, 2003. http://dx.doi.org/10.1590/ $\underline{\text { S0104-530X2003000300005 }}$

ETZKOWITZ, H.; LEYDESDORFF, L. The dynamics of innovation: from National Systems and 'Mode 2' to a Triple Helix of university-industry-government relations. Research Policy, v. 29, p. 109-123, 2000.

FLORIDA, R. The role of the university: leveraging talent, not technology. Issues in Science and Technology On Line, 1999.

GOMES, E.J. A relação universidade empresa no Brasil: testando hipóteses a partir do caso Unicamp. 2001. Tese (Doutorado) - Instituto de Geociências, Universidade Estadual de Campinas, Campinas, 2001. 
INSTITUTO BRASILEIRO DE GEOGRAFIA E ESTATÍSTICA (IBGE). Sistema Nacional de Índices de Preços ao Consumidor. Rio de Janeiro: IBGE, 2018. Disponível em: $\quad<$ https://ww2.ibge.gov.br/home/estatistica/indicadores/precos/inpc ipca/defaultinpc.

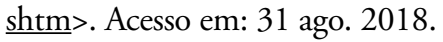

KERR, C. The uses of the University. Londres: Oxford University Press, 1972.

MOLLAS-GALLART, J. et al. Measuring third stream activities: final report to the Russel Group of Universities. Brighton: SPRU, University of Sussex, 2002.

MOWERY, D. C.; SAMPAT, B. N. Universities in national innovation systems. In: FAGERBERG, J.; MOWERY, D. C.; NELSON, R. R. (orgs.). The Oxford handbook of innovation. Oxford: Oxford University Press, 2004.

PAVITT, K. Public policies to support basic research: What can the rest of the world learn from US theory and practice? (and what they should not learn). Industrial and Corporate Change, v. 10, p. 761-779, 2001. https://doi.org/10.1093/icc/10.3.761

RASMUNSSEN, W. D. Taking the university the people: seventy-five years of cooperative extension. Ames: Iowa State University Press, 1989. Disponível em: $<$ http://www.scielo.br/scielo.php?script=sci_nlinks\&ref=000147\&pid=S0034737X201000030000300013\&lng=en>. Acesso em: $1^{\circ}$ fev. 2016.

SÁBATO, J. A.; BOTANA, N. La ciencia y la tecnología en el desarrollo futuro de América Latina. In: SÁBATO, J. A.; BOTANA, N. (orgs.). El pensamiento latinoamericano en la problemática ciencia-tecnologia-desarrollo-dependencia. Buenos Aires: Biblioteca Nacional, 2011. Disponível em: <www.mincyt.gob.ar/publicaciones > . Acesso em: 30 abr. 2014.

SAKASHITA, C. N. Caracterização das formas de intervençáo da fundaçáo junto à universidade: problemas, dilemas e soluçóes. Dissertação (Mestrado) - Faculdade de Educação, Universidade Estadual de Campinas, Campinas, 2011.

SUZIGAN, W.; ALBUQUERQUE, E. A interação entre universidades e empresas em perspectivas históricas no Brasil. In: SUZIGAN, W.; ALBUQUERQUE, E. M.; CARIO, S. A. F. (orgs.). Em busca da Inovaçáo: Interação universidade-empresa no Brasil. Belo Horizonte: Autêntica, 2011.

UNIVERSIDADE ESTADUAL DE CAMPINAS (UNICAMP). Diretoria Geral da Administração. Instruçáo DGA, no 5 de 11 de julho de 2001. Campinas: Unicamp, 2001. Disponível em: <https://www.dga.unicamp.br/Conteudos/Legislacao/ InstrucoesNormativasDGA/Instrucao DGA n 005 2001.pdf $>$. Acesso em: 15 mar. 2018.

Recebido em 30 de novembro de 2017.

Aceito em 25 de julho de 2018.

(C) 2019 Centro de Estudos Educação e Sociedade - CEDES Este é um artigo de acesso aberto distribuído nos termos de licença Creative Commons. 